\title{
Exact Cnoidal Solutions of the Extended KdV Equation
}

\author{
E. Infeld ${ }^{a}$, A. KARCZewska ${ }^{b}$, G. Rowlands ${ }^{c}$ And P. RozmeJ ${ }^{d, *}$ \\ ${ }^{a}$ National Centre for Nuclear Research, Hoża 69, 00-681 Warszawa, Poland \\ ${ }^{b}$ Faculty of Mathematics, Computer Science and Econometrics, University of Zielona Góra, \\ Z. Szafrana 4a, 65-246 Zielona Góra, Poland \\ ${ }^{c}$ Department of Physics, University of Warwick, Coventry, CV4 7AL, UK \\ ${ }^{d}$ Institute of Physics, Faculty of Physics and Astronomy, University of Zielona Góra, \\ Z. Szafrana 4a, 65-246 Zielona Góra, Poland
}

(Received February 19, 2018)

Dedicated to the memory of Dr John Dougherty (1935-2015)

\begin{abstract}
The KdV equation can be derived within the shallow water limit of the Euler equations. Over the last few decades, this equation has been extended to include both higher order effects (KdV2) and an uneven river bottom. Although this equation is not integrable and has only one conservation law, exact periodic and solitonic solutions exist for the even bottom case. The method used to find them assumes the same functional forms as for KdV solutions. The KdV2 equation imposes more constraints on the parameters of solutions. Quite unexpectedly, we found two regions in $m$ parameter space for periodic solutions. For the range of $m$ close to one the cnoidal waves are upright as expected, but are inverted in the $m$ region close to zero which is a completely new feature. The properties of exact solutions for KdV and KdV2 are compared. Numerical evolution of all the discussed exact solutions to KdV2 is stable and confirms the properties of the analytic solutions.
\end{abstract}

DOI: 10.12693/APhysPolA.133.1191

PACS/topics: shallow water waves, extended KdV equation, analytic solutions, inverted cnoidal waves

\section{Introduction}

One hundred and seventy years ago, Stokes pointed out that waves described by nonlinear models can be periodic [1]. Although several related results followed, it took half a century before the Korteveg-de Vries equation became widely known [2]. A competitive equation, Boussinesq, was formulated in 1871. It is also the theme of several recent papers [3, 4]. Another direction research has gone in it including perpendicular dynamics in $\mathrm{KdV}$, e.g. [5].

The KdV equation is one of the most ubiquitous physical equations. It consists of the mathematically simplest possible terms representing the interplay of nonlinearity and dispersion. This simplicity may be one of the reasons for success. Here we investigate this equation improved as derived from the Euler inviscid and irrotational water equations.

Just as for conventional $\mathrm{KdV}$, two small parameters are assumed: wave amplitude/depth $a / H$ and depth/wavelength squared $(H / l)^{2}$. These dimensionless expansion constants are called $\alpha$ and $\beta$. We take expansion one order higher. The new terms will then be of second order. This procedure limits considerations to waves for which the two parameters are small and comparable. Unfortunately, some authors tend to be careless about this limitation.

*corresponding author; e-mail: p.rozmej@if.uz.zgora.pl
The next approximation to Euler's equations for long waves over a shallow riverbed is ( $\eta$ is the elevation above a flat surface divided by $H$ ):

$$
\begin{aligned}
& \eta_{t}+\eta_{x}+\frac{3}{2} \alpha \eta \eta_{x}+\frac{1}{6} \beta \eta_{3 x}-\frac{3}{8} \alpha^{2} \eta^{2} \eta_{x} \\
& \quad+\alpha \beta\left(\frac{23}{24} \eta_{x} \eta_{2 x}+\frac{5}{12} \eta \eta_{3 x}\right)+\frac{19}{360} \beta^{2} \eta_{5 x}=0 .
\end{aligned}
$$

In (1) and subsequently we use low indexes for derivatives $\left(\eta_{n x} \equiv \frac{\partial^{n} \eta}{\partial x^{n}}\right)$. This second order equation was called by Marchant and Smyth [6, 7] the extended $K d V$. It was also derived in a different way in $[8]$ and $[9,10]$. We call it KdV2. It is not integrable. Not only is KdV2 non integrable, it only seems to have one conservation law (volume or mass) [11, 12]. A simple derivation of adiabatically conserved quantities can be found in [13].

Although by some appropriate scaling KdV2 can be written in a simpler form (e.g. [14, 15]) we consider solutions to the KdV2 in the form (1) for the following reasons. KdV2 is a particular case of a more general equation derived by us in the second order perturbation approach to the Euler equations for the shallow water problem with uneven bottom $[9,10]$. This equation (see, e.g., Eq. (35) in [9], Eq. (18) in [10] or Eq. (1) in [16]) contains direct terms from bottom changes and was derived in the second order perturbation approach with the assumption that the third small parameter $\delta$ is of the same order as $\alpha, \beta$. This parameter is defined as the ratio of bottom function amplitude to the mean water depth. Since we use the solutions to KdV2 as initial conditions to calculate numerical evolution of waves entering the regions where bottom changes occur we prefer to use the KdV2 equation in the form (1). 
Many authors, e.g. [17, 18], argue that equations like (1) can be transformed to an asymptotically equivalent integrable form. The asymptotic equivalence means that solutions of these equations converge to the same solution when small parameters tend to zero. This approach was first introduced with near-identity transformation (NIT) by Kodama [19] and then used and generalized by many others, e.g. [20-22]. However, NIT is an approximation in which terms of higher order are neglected. Therefore, for finite values of small parameters $(\alpha, \beta)$, solutions of NIT-transformed integrable equation are not the same as exact solutions.

The KdV equation, despite its success, is not a law of nature. It is only an approximation of the first order perturbation approach to the set of the Euler equations. However, many authors seem to forget that applicability of $\mathrm{KdV}$ is limited to $\alpha \approx \beta \ll 1$ and use it outside this range. Some authors of mathematical papers on $\mathrm{KdV}$ ignore constraints on $\mathrm{KdV}$ solutions which come from the physical meaning of dimensionless variables.

The aim of this paper is twofold. First, we present exact solutions to the KdV2 Eq. (1), which, as obtained in the second order perturbation approach, should be applicable to much larger values of $\alpha, \beta$. Second, we discuss the consequences of the constraints imposed on the coefficients of exact solutions. The results of this paper complete our studies on the hypothesis that KdV2 possesses exact solutions of the same functional forms as $\mathrm{KdV}$ but with altered coefficients. In Ref. [10] we have found exact soliton solutions for $\mathrm{KdV} 2$ in the form $\eta(x, t)=A \operatorname{sech}(B(x-v t))^{2}$. In Refs. [23, 24] we have constructed exact periodic solutions to $\mathrm{KdV} 2$ in the form of superpositions $\eta_{ \pm}(x, t)=\frac{A}{2}\left[\operatorname{dn}[B y, m]^{2} \pm \sqrt{m} \operatorname{cn}[B y, m] \operatorname{dn}[B y, m]\right]+D$, where $y=x-v t$. In this paper we have found the cnoidal solutions to $\mathrm{KdV} 2$ in the form $\eta(x, t)=A \operatorname{cn}[B(x-$ $v t), m]^{2}+D$ which have two branches. In the first one, when the parameter $m$ is close to 1 , the usual cnoidal waves similar to those which are solutions to $\mathrm{KdV}$ are obtained. In the second, when the parameter $m$ is close to 0 , the new, inverted cnoidal solutions are obtained. In this case $\mathrm{KdV}$ fails since its solutions correspond for $m \rightarrow 0$ to very short waves which is in contradiction to the long wave assumption basic for derivation of $\mathrm{KdV}$ and KdV2. The inverted cnoidal solution of KdV2 found in this paper is free of this contradiction.

The paper is organised as follows. In Sect. 2 standard derivations of solitonic and periodic solutions to $\mathrm{KdV}$ are reviewed, followed by a description of an algebraic approach to KdV. In Sect. 3 the exact soliton solution to KdV2 [10] is recalled with an additional constraint on the dimensionless amplitude of the soliton. Exact periodic solutions to $\mathrm{KdV} 2$ in the form of $\mathrm{cn}^{2}$ cnoidal functions are derived in Sect. 4. Quite unexpectedly two branches of solutions are found. Numerical evolution of several examples of different solutions to KdV2 are presented in Sect. 5. Section 6 contains conclusions.

\section{KdV solutions}

For further discussions of new properties of KdV2 solutions we first briefly remind $\mathrm{KdV}$ solutions.

\subsection{Standard approach}

$\mathrm{KdV}$ equation is given by the first line of (1). For standard approach to derive exact solutions, see, e.g. [25, 26]. Here we will remind only some steps of these derivations and the final results. Introducing new variable $\xi=x-c t$, where $c=1+\alpha c_{1}$ and dividing $\mathrm{KdV}$ equation by $\alpha$ one obtains an ODE equation

$$
-c_{1} \eta_{\xi}+\frac{3}{2} \eta \eta_{\xi}+\frac{1}{6} \frac{\beta}{\alpha} \eta_{3 \xi}=0
$$

Integration gives ( $r$ is an integration constant)

$$
-c_{1} \eta+\frac{3}{4} \eta^{2}+\frac{1}{6} \frac{\beta}{\alpha} \eta_{2 \xi}=\frac{1}{4} r
$$

Then multiplication by $\eta_{\xi}$ and next integration yields

$$
\frac{1}{3} \frac{\beta}{\alpha}\left(\eta_{\xi}\right)^{2}=-\eta^{3}+2 c_{1} \eta^{2}+r \eta+s=: f(\eta),
$$

where $s$ is another integration constant. Then if solutions are such that $\eta(\xi) \rightarrow 0$ when $\xi \rightarrow \pm \infty$ then $r=s=0$. In this case $f(\eta)=\eta^{2}\left(2 c_{1}-\eta\right)$ and integration of (4) is easily obtained with hyperbolic functions leading finally to single soliton solution

$$
\eta(x, t)=A \operatorname{sech}^{2}\left[\sqrt{\frac{3 \alpha}{4 \beta} A}\left(x-t\left(1+\frac{\alpha}{2} A\right)\right)\right] .
$$

The amplitude $A$ can, in principle, be arbitrary as long as this is not in contradiction to the basic assumptions for derivation of $\mathrm{KdV}$.

The path to obtain exact periodic solutions is much more involved. The most detailed discussion of this problem is contained in [26]. Below, we remind only few important steps and formulae. In general, integration constants can be nonzero. Then, assuming that $\eta_{1}<\eta_{2}<\eta_{3}$ are roots of polynomial $f(\eta)$, the polynomial can be written as

$$
f(\eta)=-\left(\eta-y_{1}\right)\left(\eta-y_{2}\right)\left(\eta-y_{3}\right) .
$$

Real-valued solutions are possible when $-\eta_{2} \leq \eta \leq \eta_{1}$, only. Then solution of (4) can be found in the form

$$
\eta(\xi)=\eta_{1} \cos ^{2} \chi(\xi)-\eta_{2} \sin ^{2} \chi(\xi) .
$$

Then (4) takes form

$$
\frac{4 \beta}{3 \alpha} \chi_{\xi}^{2}=\left(\eta_{1}+\eta_{3}\right)-\left(\eta_{1}+\eta_{2}\right) \sin ^{2} \chi .
$$

Denoting $m=\frac{\eta_{1}+\eta_{2}}{\eta_{1}+\eta_{3}} \in[0,1]$ and $\Delta^{2}=\frac{4 \beta}{3 \alpha\left(\eta_{1}+\eta_{3}\right)}$ one obtains from (4):

$$
\Delta^{2} \chi_{\xi}^{2}=1-m \sin ^{2} \chi
$$

Integration yields

$$
\frac{1}{\Delta} \int_{0}^{\xi} \mathrm{d} \hat{\xi}=\mp \int_{0}^{\chi} \frac{\mathrm{d} \hat{\chi}}{\sqrt{1-m \sin ^{2} \hat{\chi}}} \Longrightarrow \pm \frac{\xi}{\Delta}=F(\chi \mid m),
$$

where $F(\chi \mid m)$ is the incomplete elliptic integral of the first kind. Since the inverse functions are

$$
\cos \chi=\operatorname{cn}\left(\frac{\xi}{\Delta} \mid m\right), \quad \sin \chi=\operatorname{sn}\left(\frac{\xi}{\Delta} \mid m\right),
$$


then from (8) solution is obtained in the form

$$
\eta(\xi)=-\eta_{2}+\left(\eta_{1}+\eta_{2}\right) \mathrm{cn}^{2}\left(\frac{\xi}{\Delta} \mid m\right) .
$$

In the next steps Dingemans [26] stresses the conditions which supply additional relations between $\eta_{1}, \eta_{2}, \eta_{3}$. The first one is given by the periodicity of the $\mathrm{cn}^{2}$ function. The second condition requires that the mean free surface elevation should coincide with the still water surface and express the fluid's incompressibility.

\subsection{Algebraic approach}

From theory of nonlinear differential equation it is known, see, e.g. [27-30], that for some classes of such equations exact solutions should exist in forms of either hyperbolic or the Jacobi elliptic functions. It appears that both $\mathrm{KdV}$ and $\mathrm{KdV} 2$ equations belong to these classes. Therefore one can directly look for solutions of these equations assuming a particular form of solution. Our main goal is to find exact solutions of $\mathrm{KdV} 2$ equation. In order to introduce the reader to the algebraic approach we begin with much simpler KdV case.

\subsubsection{Single soliton solution}

Soliton solution is assumed as (amplitude is set equal to 1$)$ :

$$
\eta(x, t)=A \operatorname{sech}^{2}[B(x-v t)]=A \operatorname{sech}^{2}(B y),
$$

where $y=x-v t$. Substitution (13) into KdV (see Eq. (36) limited to the first three terms) gives

$-\frac{1}{3} A B \tanh (B x) \operatorname{sech}^{4}(B x)\left[G_{0}+G_{1} \cosh (2 B x)\right]$.

Equation (14) is valid for any argument only when simultaneously

$$
\begin{aligned}
& G_{0}=3-3 v+9 \alpha A-10 \beta B^{2}=0, \\
& G_{1}=3-3 v+2 \beta B^{2}=0 .
\end{aligned}
$$

This gives immediately

$$
B^{2}=\frac{3 \alpha}{4 \beta} A, \quad v=1+\frac{\alpha}{2} A,
$$

and solution coincides with (5).

Remark: It is clear from (17) that solutions exist for arbitrary parameters $\alpha, \beta$, provided both are small.

\subsubsection{Periodic solution}

In this case solution is postulated in the form of cnoidal wave

$$
\eta(x, t)=A \mathrm{cn}^{2}(B(x-v t), m)+D .
$$

[Equivalently, instead of Jacobi elliptic cn function, $\mathrm{dn}$ or sn Jacobi elliptic functions can be used.]

Note that the form (18) is identical with (12) when $A=\eta_{1}+\eta_{2}$ and $D=-\eta_{2}$.

Then, substitution of (18) into $\mathrm{KdV}$ yields equation analogous to (14):

$$
\frac{1}{3} A B \text { cn sn dn }\left(G_{0}+G_{1}(c n)^{2}\right)=0 .
$$

So, there must be

$$
G_{0}=4 \beta B^{2}-8 \beta B^{2} m-9 \alpha D+6 v-6=0,
$$

$$
G_{1}=12 \beta B^{2} m-9 \alpha A=0 .
$$

Equation (21) implies

$$
B^{2}=\frac{3 \alpha}{4 \beta} \frac{A}{m} .
$$

Volume conservation condition (details will be explained later) determines

$$
D=-\frac{A}{m}\left(\frac{E(m)}{K(m)}+m-1\right) .
$$

In formula (23), $E(m)$ and $K(m)$ are the complete elliptic integral and the complete elliptic integral of the first kind, respectively. Then from (20) one has

$$
v=1+\frac{\alpha A}{2 m}\left(2-m-3 \frac{E(m)}{K(m)}\right) .
$$

Denoting

$$
E K(m):=2-m-3 \frac{E(m)}{K(m)}
$$

one obtains

$$
\begin{aligned}
& D=\frac{A}{3 m}[E K(m)-2 m+1], \\
& v=1+\frac{\alpha A}{2 m} E K(m) .
\end{aligned}
$$

The function $E K(m)$ (see Fig. 1) is equal to zero for

$$
m=m_{s} \approx 0.9611494753812
$$

and reaches the value 1 for $m=1$.

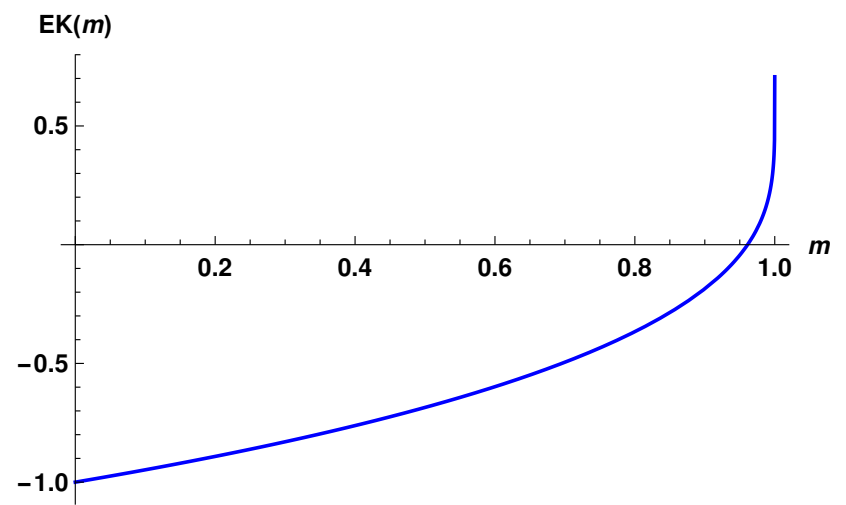

Fig. 1. Plot of the function $E K(m)(25)$.

The limit $m \rightarrow 1$ gives the single soliton solution discussed in previous subsection.

It is well known, see e.g. [31, 32], that cnoidal solutions of $\mathrm{KdV}$ are not good approximation for short shallow water waves. The limit $m \rightarrow 0$ preserves finite $B$ (or finite wavelength $\sim 1 / B)$ in $(22)$ when the amplitude $A$ is proportional to $m$, only, that is, for infinitesimal waves. In reverse, if $A$ is finite then for $m \rightarrow 0$ or the wavelength tends to zero (since $B \rightarrow \infty$ ). At the same time velocity (27) tends to minus infinity, see Fig. 2.

\section{Exact single soliton solution for $\mathrm{KdV2}$}

In Ref. [10] we found exact single solution for $\mathrm{KdV} 2$ assuming the same form of the solution as for $\mathrm{KdV}$, that 


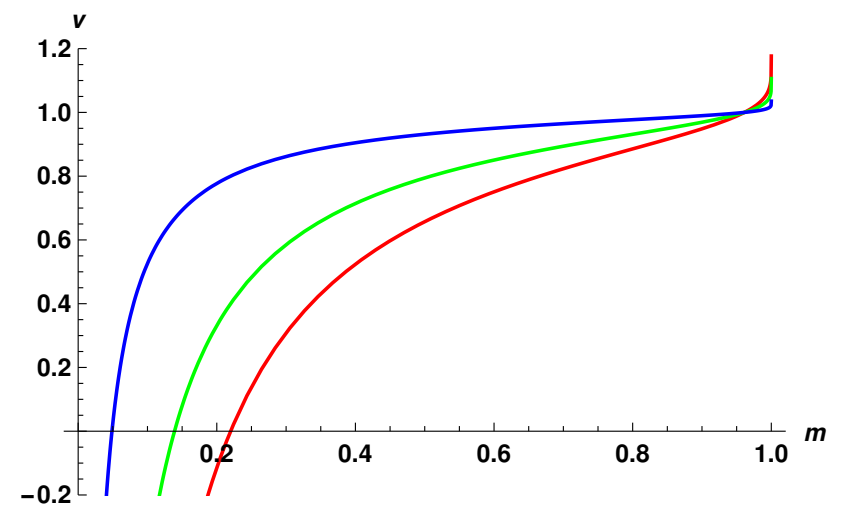

Fig. 2. Velocity (27) of KdV periodic solution (18) as function of $m$ for $\alpha=0.5,0.3,0.1$ plotted with red, green and blue lines, respectively. Coefficient $A=1$.

is (13). Below we briefly remind that result. Insertion of (13) into (1) gives (after some simplifications) equation analogous to (14):

$$
C_{0}+C_{2} \operatorname{sech}^{2}(B y)+C_{4} \operatorname{sech}^{4}(B y)=0,
$$

which supplies three conditions on parameters of solution formula

$$
\begin{aligned}
& C_{0}=(1-v)+\frac{2}{3} B^{2} \beta+\frac{38}{45} B^{4} \beta^{2} \\
& C_{2}=\frac{3 A \alpha}{4}-B^{2} \beta+\frac{11}{4} A \alpha B^{2} \beta-\frac{19}{3} B^{4} \beta^{2} \\
& C_{4}=-\left(\frac{1}{8}\right)(A \alpha)^{2}-\frac{43}{12} A \alpha B^{2} \beta+\frac{19}{3} B^{4} \beta^{2} .
\end{aligned}
$$

From Eq. (31), denoting $z=\frac{\beta B^{2}}{\alpha A}$ we obtain

$$
\frac{19}{3} z^{2}-\frac{43}{12} z-\frac{1}{8}=0
$$

with roots

$$
\begin{aligned}
& z_{1}=\frac{43-\sqrt{2305}}{152} \approx-0.033<0, \\
& z_{2}=\frac{43+\sqrt{2305}}{152} \approx 0.599>0 .
\end{aligned}
$$

Inserting $\beta B^{2}=\alpha A z$ into (30) we have:

$$
A=\frac{z-\frac{3}{4}}{\alpha z\left(\frac{11}{4}-\frac{19}{3} z\right)}>0
$$

for both $z_{1}$ and $z_{2}$.

Since $z_{1}<0$ thus $B$ is real only when $z=z_{2}$, so in this case

$$
\begin{aligned}
& A=\frac{0.242399}{\alpha}, \\
& B^{2}=\frac{\alpha}{\beta} A z_{2}=\approx 0.599 \frac{\alpha}{\beta} A \approx \frac{0.145137}{\beta} .
\end{aligned}
$$

Then Eq. (29) determines velocity

$$
v=1+\frac{2}{3} \alpha A z_{2}+\frac{38}{45}\left(\alpha A z_{2}\right)^{2} \approx 1.11455 .
$$

These results are the same as in Sect. 4 of [10].
Comparing single soliton solutions for $\mathrm{KdV}$ and $\mathrm{KdV} 2$ we see the following differences:

- For $\operatorname{KdV} B=\sqrt{0.75 \frac{\alpha}{\beta}}, \quad$ for $\operatorname{KdV} 2 B \approx \sqrt{0.6 \frac{\alpha}{\beta}}$. This difference in $B$ values means that the KdV2 soliton is a little wider than that of $\mathrm{KdV}$ (for the same parameters $\alpha, \beta$ ), see Fig. 3 .

- For $\mathrm{KdV} v=1+\frac{\alpha}{2} A$, for $\mathrm{KdV} 2 v \approx 1.11455$ is fixed.

- $\mathrm{KdV}$ admits a one parameter family of solutions (for instance A can be arbitrary). KdV2 imposes one more condition on coefficients of the solution, therefore parameters $\alpha, \beta$ of the equation, determine a single solution with parameters given by Eqs. (34)-(35). Such kind of fixed soliton solutions are sometimes called embedded solitons [16].

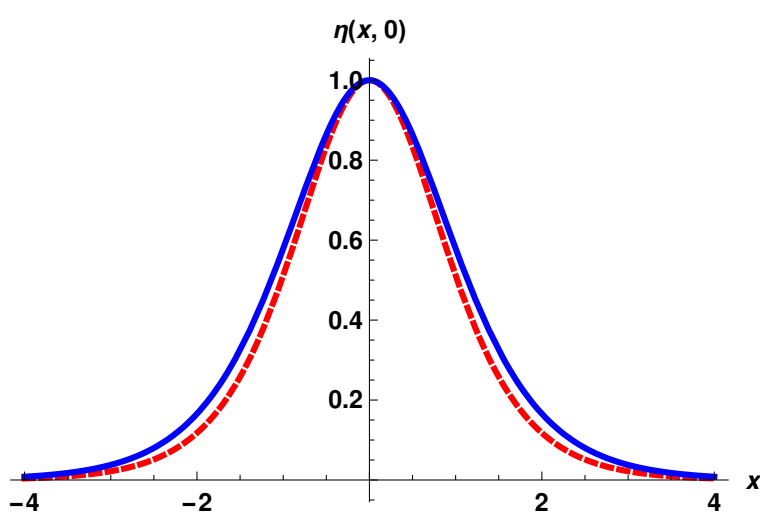

Fig. 3. Comparison of the profile of KdV soliton - red dashed line with KdV2 soliton - blue line. Both curves are obtained for the same value of the amplitude $A=1$.

\section{Exact periodic solutions for KdV2}

We look for periodic nonlinear wave solutions of KdV2 (1). Introduce $y=x-v t$. Then $\eta(x, t)=\eta(y)$, $\eta_{t}=-v \eta_{y}$ and Eq. (1) takes the form of an ODE

$$
\begin{aligned}
& (1-v) \eta_{y}+\frac{3}{2} \alpha \eta \eta_{y}+\frac{1}{6} \beta \eta_{3 y}-\frac{3}{8} \alpha^{2} \eta^{2} \eta_{y} \\
& \quad+\alpha \beta\left(\frac{23}{24} \eta_{y} \eta_{2 y}+\frac{5}{12} \eta \eta_{3 y}\right)+\frac{19}{360} \beta^{2} \eta_{5 y}=0 .
\end{aligned}
$$

Now assume the periodic solution to be in the same form as corresponding solution of $\mathrm{KdV}$

$$
\eta(y)=A \mathrm{cn}^{2}(B y, m)+D,
$$

where $A, B, D$ are yet unknown constants ( $m$ is the elliptic parameter). The constant $D$ must ensure that the volume of water is the same for all $m$.

Now we calculate all derivatives $\eta_{n y}$ entering (36). Using properties of the Jacobi elliptic functions and their derivatives one can express them as functions of $\mathrm{cn}^{2}$. So 
$\eta_{y}=2 A B \operatorname{cn}[-\mathrm{sn} \mathrm{dn}]=-2 B \mathrm{cn} \operatorname{sn} \mathrm{dn}$,

$\eta_{2 y}=2 A B^{2}\left[1-m+(4 m-2) \mathrm{cn}^{2}-3 m \mathrm{cn}^{4}\right]$,

$\eta_{3 y}=8 A B^{3} \mathrm{cn} \operatorname{dn} \operatorname{sn}\left[1-2 m+3 m \mathrm{cn}^{2}\right]$,

$\eta_{5 y}=-16 A B^{5} \mathrm{cn} \operatorname{dn} \operatorname{sn}\left[\left(2-17 m+17 m^{2}\right)\right.$

$$
\left.+\left(30 m-60 m^{2}\right) \mathrm{cn}^{2}+45 m^{2} \mathrm{cn}^{4}\right] .
$$

Denote (36) as

$$
E_{1}+E_{2}+E_{3}+E_{4}+E_{5}+E_{6}+E_{7}=0,
$$

where $($ common factor CSD $=(-2 A B$ cn sn dn $))$ :

$$
\begin{aligned}
E_{1} & =(1-v) \eta_{y}=(1-v) \mathrm{CSD}, \\
E_{2} & =\frac{3}{2} \alpha \eta \eta_{y}=\frac{3}{2} \alpha\left(\mathrm{cn}^{2}+D\right) \mathrm{CSD}, \\
E_{3} & =\frac{1}{6} \beta \eta_{3 x}=-\frac{2}{3} \beta B^{2}\left[1-2 m+3 m \mathrm{cn}^{2}\right] \mathrm{CSD}, \\
E_{4} & =-\frac{3}{8} \alpha^{2} \eta^{2} \eta_{y}=-\frac{3}{8} \alpha^{2}\left(\mathrm{cn}^{2}+D\right)^{2} \mathrm{CSD}, \\
E_{5} & =\frac{23}{24} \alpha \beta \eta_{y} \eta_{2 y}= \\
\frac{23}{12} & \alpha \beta B^{2}\left[1-m+(4 m-2) \mathrm{cn}^{2}-3 m \mathrm{cn}^{4}\right] \mathrm{CSD}, \\
E_{6} & =\frac{5}{12} \alpha \beta \eta \eta_{3 y}= \\
\quad & \frac{5}{3} \alpha \beta B^{2}\left(\mathrm{cn}^{2}+D\right)\left[1-2 m+3 m \mathrm{cn}^{2}\right] \mathrm{CSD}, \\
E_{7} & =\frac{19}{360} \beta^{2} \eta_{5 x}=\frac{19}{45} \beta^{2} B^{4}\left[\left(2-17 m+17 m^{2}\right)\right. \\
& \left.+\left(30 m-60 m^{2}\right) \mathrm{cn}^{2}+45 m^{2} \mathrm{cn}^{4}\right] \mathrm{CSD}
\end{aligned}
$$

Then (42) becomes

$$
(-2 B \mathrm{cn} \text { sn } \mathrm{dn})\left[F_{0}+F_{1} \mathrm{cn}^{2}+F_{2} \mathrm{cn}^{4}\right]=0 .
$$

Equation (50) is valid for arbitrary argument of $\mathrm{cn}^{2}$ when all three coefficients $F_{0}, F_{1}, F_{2}$ vanish simultaneously. This gives us a set of three equations for the coefficients $v, B, D$ :

$$
\begin{gathered}
F_{0}=690 \alpha A \beta B^{2}(m-1)-\left(\beta B^{2}\right)^{2}(2584 m(m-1) \\
+304)+240 \beta B^{2}(1-2 m)-60 \alpha D\left(10 \beta B^{2}(2 m-1)\right. \\
\quad+9)+135(\alpha D)^{2}+360(v-1)=0, \\
F_{1}=90 \alpha A\left[22 \beta B^{2}(1-2 m)+3 \alpha D-6\right] \\
\quad+120 \beta B^{2} m\left[38 \beta B^{2}(2 m-1)+15 \alpha D+6\right]=0,(52) \\
F_{2}=45\left(3 \alpha^{2} A^{2}+86 \alpha A \beta B^{2} m-152 \beta^{2} B^{4} m^{2}\right)=0 .
\end{gathered}
$$

Equations (51)-(53), supplemented by the volume conservation law, allow us to find all unknowns as functions of the elliptic parameter $m$. Below we show these solutions explicitly.

$$
\text { Now, denote } z=\frac{B^{2} \beta}{A \alpha} m \text {. }
$$

Then, Eq. (53) becomes identical with (32) and has the same roots (33).

\subsection{Periodicity and volume conservation}

In principle, exact periodic solutions of $\mathrm{KdV} 2$ with $D=0$ exist. They make sense from a mathematical point of view. For KdV case the derivation of such periodic solutions is presented in Whitham's book [25]. The more careful derivation, presented by Dingemans [26], stresses that periodic solutions should have profile uplifts and depressions with respect to the undisturbed water level. Therefore the volume conservation condition is crucial for obtaining proper physical solutions.

Volume conservation determines the value of $D$. Here by mass conservation we mean that for each $m$ the solution involves the same volume of water

$$
\int_{0}^{L}\left(A \operatorname{cn}^{2}(B y, m)+D\right) \mathrm{d} y=0 .
$$

Then

$$
D=-\frac{A}{L} \int_{0}^{L} \operatorname{cn}^{2}(B y, m) \mathrm{d} y \equiv-\frac{A}{L} I(L),
$$

where $L$ is the wavelength. The periodicity condition implies

$$
\mathrm{cn}^{2}(B l, m)=\mathrm{cn}^{2}(0, m) \Longrightarrow L=\frac{2 K(m)}{B},
$$

where $K(m)$ is the complete elliptic integral of the first kind. Hence

$$
\begin{aligned}
D & =-\frac{A}{L} I(L)= \\
& -\frac{[E(\operatorname{am}(2 K(m) \mid m) \mid m)+(m-1) K(m)]}{2 m K(m)},
\end{aligned}
$$

where $E(\Theta \mid m)$ is the elliptic integral of the second kind and $\operatorname{am}(x \mid m)$ is the Jacobi elliptic function amplitude. Since

$$
\frac{E(\operatorname{am}(2 K(m) \mid m) \mid m))}{2 K(m)} \equiv \frac{E(m)}{K(m)},
$$

where $E(m)$ is the complete elliptic integral, and (57) simplifies to

$$
D=-\frac{A}{m}\left[\frac{E(m)}{K(m)}+m-1\right] .
$$

The function $\left[\frac{E(m)}{K(m)}+m-1\right]$ is positive for $m \in(0,1)$ and vanishes at $m=0$ and $m=1$. For $m \rightarrow 0 \quad D$ tends to $-\frac{A}{2}$ which is in agreement with sinusoidal (cosinusoidal) limit of the solution, whereas for $m \rightarrow 1, D$ tends to 0 , the solution becomes a soliton.

\subsection{Coefficients of the exact solutions to KdV2}

Without any assumptions on $m, \alpha, \beta$, other than $0 \leq$ $m \leq 1$ we obtained the set of four conditions (51)-(53) and (59) on $A, B, D, v$ and $m$. Since Eq. (53) admits two values for $z$ then we have to consider two different cases. 
4.2.1. Case $z=z_{2}=\frac{43+\sqrt{2305}}{152}$

Solving the set (51)-(53) and (59) for $z=z_{2}$ one obtains

$$
\begin{aligned}
A & =A(m, \alpha)=\frac{3(51-\sqrt{2305}) m}{37 \alpha E K(m)} \approx \frac{0.2424}{\alpha} \frac{m}{E K(m)} \\
B & =B(m, \beta)=\sqrt{\frac{3(-14+\sqrt{2305})}{703 \beta E K(m)}}, \\
D & =D(m, \alpha)=\frac{(51-\sqrt{2305})}{37 \alpha}\left(1-\frac{2 m-1}{E K(m)}\right), \\
v & =v(m)=\frac{9439-69 \sqrt{2305}}{5476} \\
& -\frac{\left(377197-7811 \sqrt{2305)\left(m^{2}-m+1\right)}\right.}{520220 E K(m)^{2}} \approx \\
& 1.11875-0.00420523 \frac{\left(m^{2}-m+1\right)}{E K(m)^{2}} .
\end{aligned}
$$

Hence, $B$ is real-valued only when $E K(m)>0$ (see Fig. 1), that is for

$$
m>m_{s} \approx 0.9611494753812 \text {. }
$$

Therefore, for this branch of solutions with $z=z_{2}$, the elliptic parameter $m \in\left(m_{s}, 1\right]$. For $m>m_{s}$, the amplitude $A>0$.

Notice that the velocity depends only on $m$.

The dependence of $A, B, D, v$ on $m$ for several cases of $\alpha, \beta$ parameters is displayed in Figs. $4-7$.

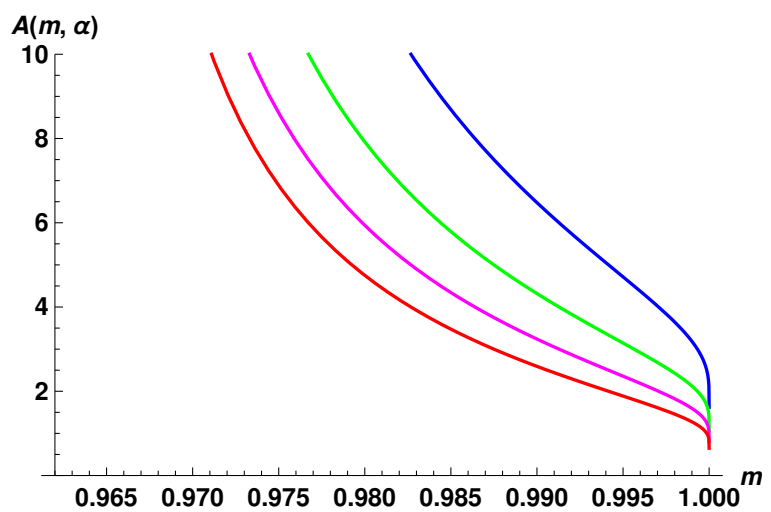

Fig. 4. Amplitude $A$ (60) as function of $m$ for $\alpha=$ $0.2,0.3,0.4,0.5$, represented by blue, green, magenta, and red lines, respectively.

Formulas (60)-(63) and Figs. 4-7 indicate that physically relevant solutions are obtained in a narrow range of $m$ close to 1 . Only for such $m$ values of $A$ are realistic (not very big). This conclusion is strengthened by the behaviour of velocity as function of $m$. Velocity is positive for $m>m_{v=0}$, where $m_{v=0} \approx 0.97357$.
In Fig. 8 profiles of cnoidal KdV solutions and KdV2 solutions are compared for $m$ close to 1 assuming the same amplitude for both solutions.

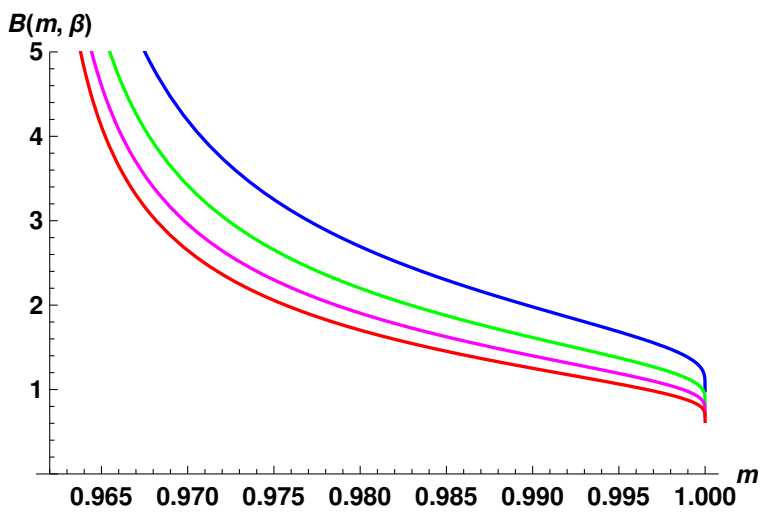

Fig. 5. Coefficient $B(61)$ as function of $m$ for $\beta=$ $0.2,0.3,0.4,0.5$, represented by blue, green, magenta, and red lines, respectively.

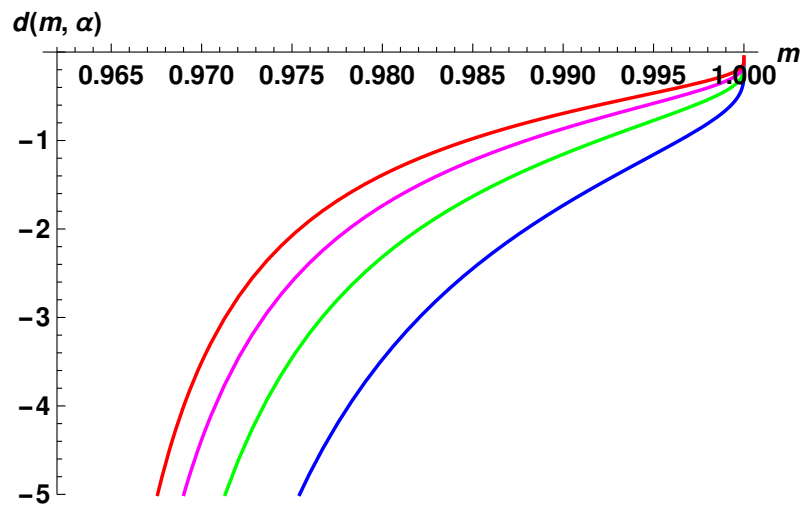

Fig. 6. Coefficient $D(62)$ as function of $m$ for $\alpha=$ $0.2,0.3,0.4,0.5$, represented by blue, green, magenta, and red lines, respectively.

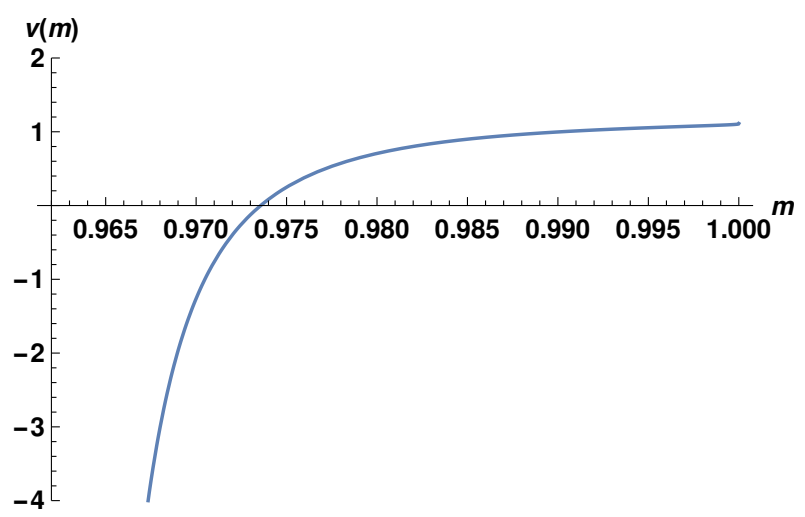

Fig. 7. Velocity $v(63)$ as function of $m$.

It is worth to emphasize that in the limit $m \rightarrow 1$ coefficients of solutions (60)-(63) receive values known for single soliton KdV2 solutions given in [10]. 


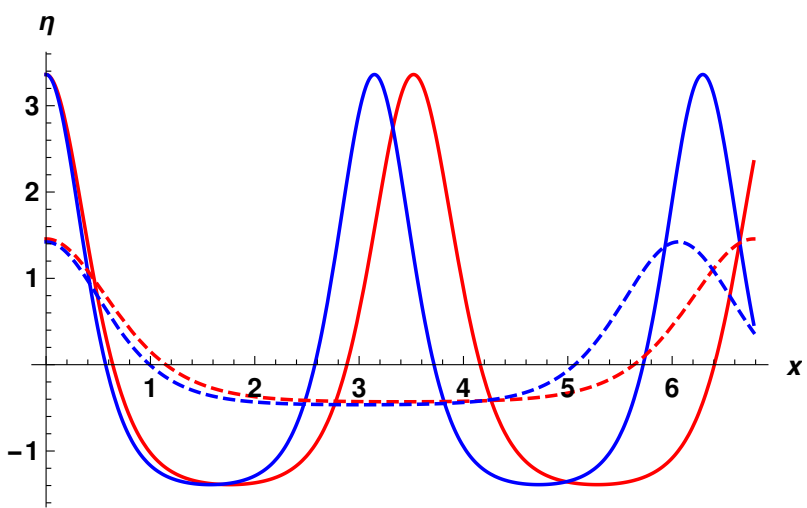

Fig. 8. Profiles of KdV2 solutions (red) and KdV solutions (blue) for case $m=0.98$ (solid) and $m=0.995$ (dashed). All profiles are obtained with $\alpha=0.5$ and $\beta=0.4$. Amplitudes of $\mathrm{KdV}$ solutions are set to be equal to amplitudes of $\mathrm{KdV} 2$ solutions.

4.2.2. Case $z=z_{1}=\frac{43-\sqrt{2305}}{152}$

Now,

$$
\begin{aligned}
A & =\frac{3(51+\sqrt{2305}) m}{37 \alpha E K(m)} \approx 8.02787 \frac{m}{\alpha E K(m)}, \\
B & =\sqrt{-\frac{3(14+\sqrt{2305})}{703 \beta E K(m)}}, \\
D & =\frac{(51+\sqrt{2305})}{37 \alpha}\left(1-\frac{2 m-1}{E K(m)}\right), \\
v & =\frac{9439+69 \sqrt{2305}}{5476} \\
& -\frac{(377197+7811 \sqrt{2305})\left(m^{2}-m+1\right)}{520220 E K(m)^{2}} \approx \\
& 2.32866-1.44594 \frac{\left(m^{2}-m+1\right)}{E K(m)^{2}} .
\end{aligned}
$$

Figures 9-12 show the dependence of $A, B, D, v$ on $m$ for several cases of $\alpha, \beta$ parameters for this branch of KdV2 solutions.

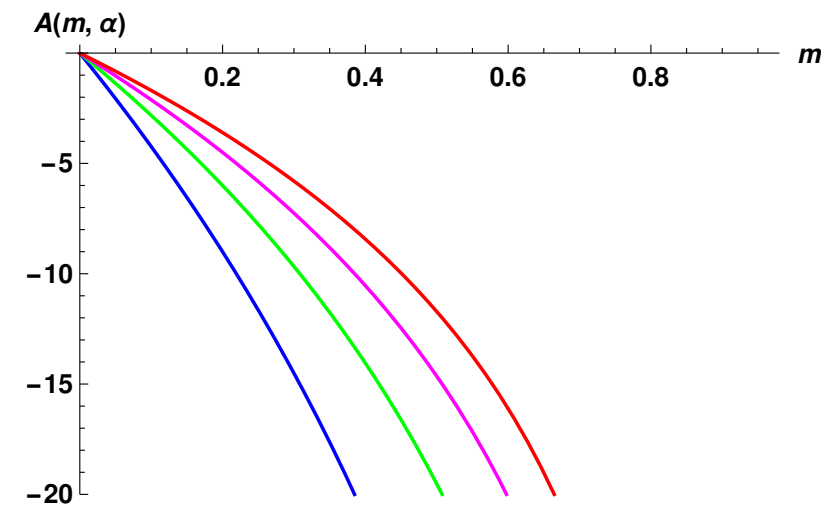

Fig. 9. Amplitude $A$ (65) as function of $m$ for $\alpha=$ $0.2,0.3,0.4,0.5$, represented by blue, green, magenta, and red lines, respectively.

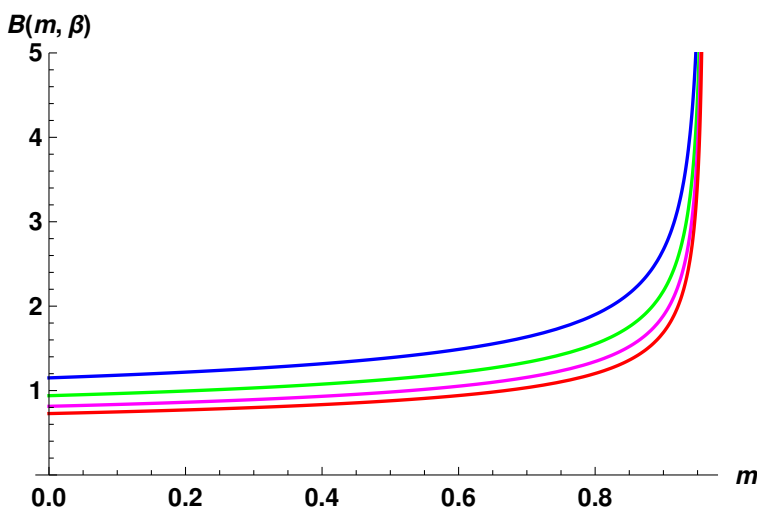

Fig. 10. Coefficient $B(66)$ as function of $m$ for $\beta=$ $0.2,0.3,0.4,0.5$, represented by blue, green, magenta, and red lines, respectively.

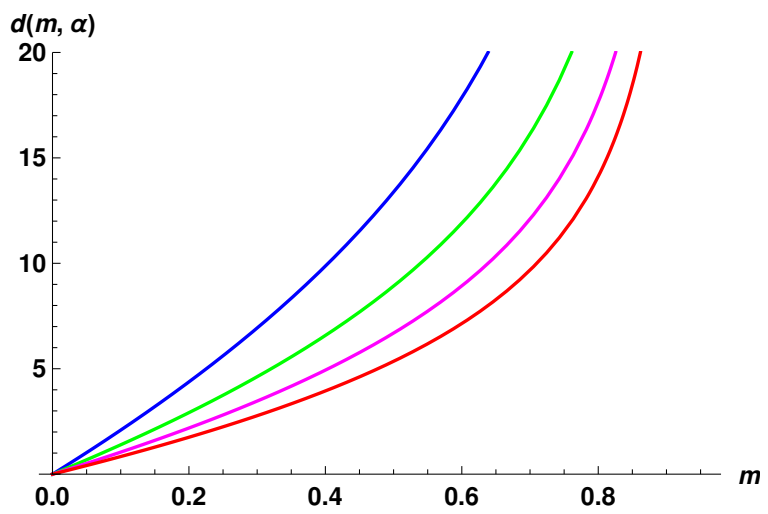

Fig. 11. Coefficient $D(67)$ as function of $m$ for $\alpha=$ $0.2,0.3,0.4,0.5$, represented by blue, green, magenta, and red lines, respectively.

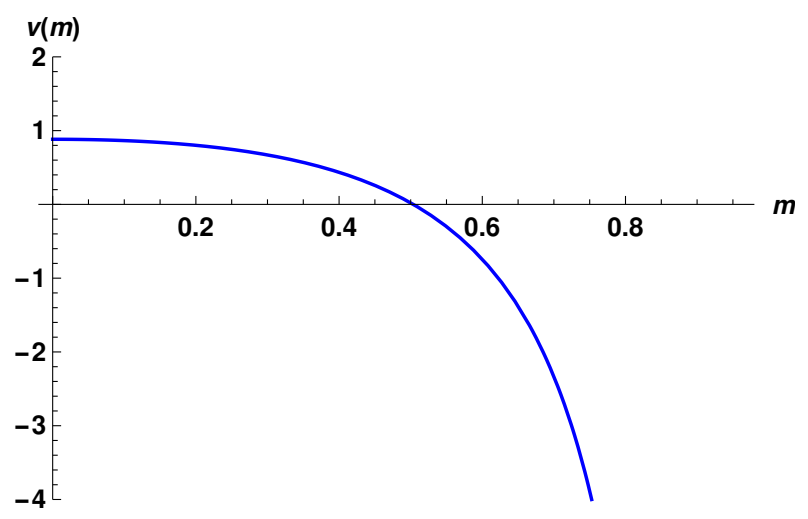

Fig. 12. Velocity $v(68)$ as function of $m$.

In this case physically reasonable values of $|A|$ occur only for $m$ close to 0 . Velocity stays positive for $m<$ $m_{v=0}$, where $m_{v=0} \approx 0.50367$. It is worth to note that since $A<0$, solutions are "inverted" cnoidal functions (with crests down and troughs up). This is completely new result. 
These new solutions are, however, not much different from usual cosine waves. In Fig. 13 the profile of the inverted cnoidal wave, obtained in this branch with $\alpha=$ $0.3, \beta=0.5$ and $m=0.2$, is compared with the cosine wave of the same amplitude and wavelength.

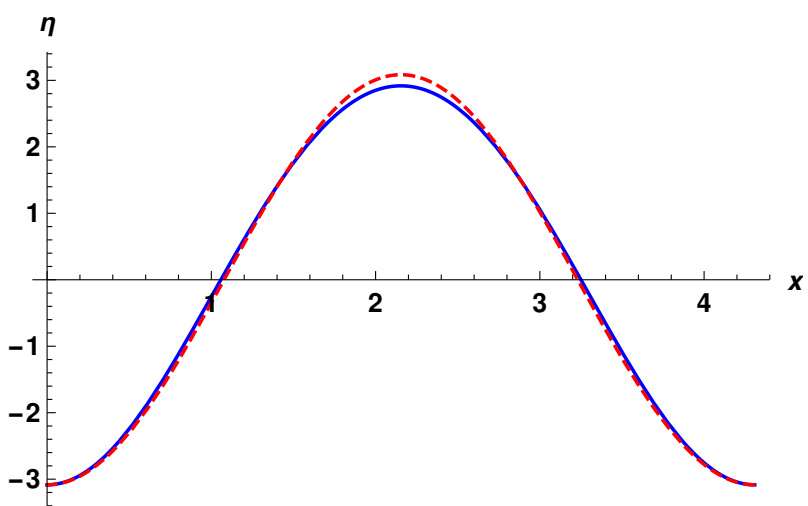

Fig. 13. Profiles of KdV2 solution (blue line) with the cosine wave of the same amplitude and wavelength (red, dashed line). The KdV2 solution corresponds to the case $\alpha=0.3, \beta=0.5$ and $m=0.2$.

\section{Numerical evolution}

In order to check our analytic results we followed numerically the evolution of several cnoidal waves. We used the finite difference (FDM) code developed for KdV2 in fixed frame (1) in our previous papers $[9,10]$. In examples presented in this subsection we assume the initial wave to be the exact cnoidal wave $\eta(x, t)=A \mathrm{cn}^{2}[B(x-$ $v t), m]+D$. The algorithm used was the ZabuskyKruskal one [33], modified in order to include additional terms. The space derivatives of $\eta(x, t)$ were calculated numerically step by step from the grid values of the function and lower order derivatives by a nine-point central difference formula. Calculations were performed on the interval $x \in[0, \lambda]$ with periodic boundary conditions of $N$ grid points. The time step $\Delta t$ was chosen as in [33], i.e., $\Delta t \leq(\Delta x)^{3} / 4$. The calculations shown in this paper used grids with $N=200$. In calculations presented below the number of time steps reached $10^{7}--10^{8}$. In all cases the algorithm secures volume (mass) conservation up to 10-11 decimal digits. The precision of our model was confirmed in our studies with the finite element method (FDM) [34, 35].

An example of the motion of the normal cnoidal wave, the solution of the KdV2 equation, obtained with numerical evolution for $\alpha=0.5, \beta=0.4, m=0.995$ is shown in Fig. 14. This is the same wave as that shown in Fig. 8 with the red dashed line.

The numerical solutions of normal cnoidal waves obtained for the $z=z_{1}$ branch are stable. The profile shown by the open symbols in Fig. 14, obtained after $2.4 \times 10^{7}$ time steps deviates from the analytic result by less than $10^{-5}$. Other tests made with initially perturbed solutions

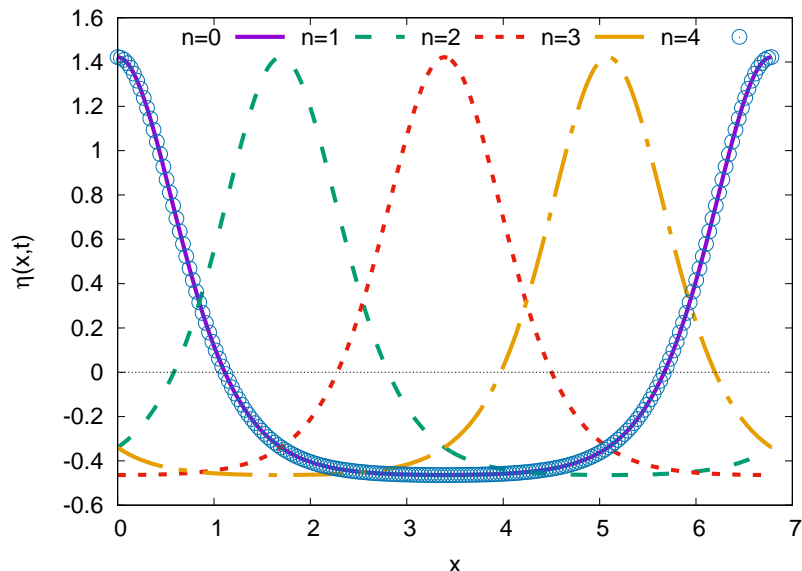

Fig. 14. Time evolution of the normal cnoidal wave for the case of parameters $\alpha=0.5, \beta=0.4, m=0.995$. Profiles are displayed at time instants $t_{n}=n \mathrm{~d} t$, where $\mathrm{d} t=\frac{1}{4} T$.

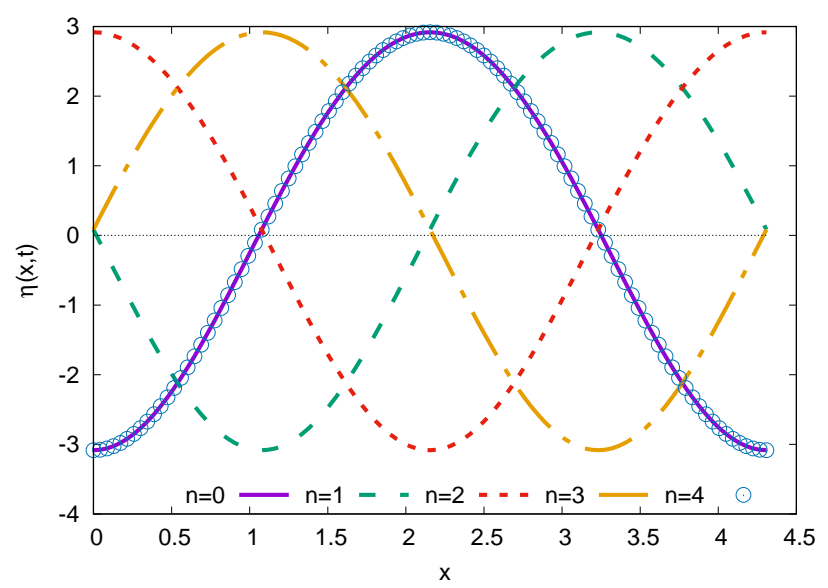

Fig. 15. Time evolution of the inverted cnoidal wave i for the case $\alpha=0.3, \beta=0.5$ and $m=0.2$. Profiles are displayed at time instants $t_{n}=n \mathrm{~d} t$, where $\mathrm{d} t=\frac{1}{4} T$.

confirm their numerical stability. In these tests analytic solutions (37) were perturbed by a cosine wave with the amplitude of $1 \%$ of the cnoidal wave amplitude. Profiles obtained after one period overlapped the initial profiles within the line width. Numerical solutions are stable for much longer time intervals, as well.

The same stability of numerical solutions is obtained for inverted cnoidal waves. An example of the motion of the inverted cnoidal solution to the KdV2 for $z=z_{1}$ branch, obtained by numerical evolution, is presented in Fig. 15. The displayed case corresponds to the wave with $\alpha=0.3, \beta=0.5$ and $m=0.2$. This is the same wave as that displayed with the solid line in Fig. 13. The deviations of the profile obtained by the numerical evolution of the inverted cnoidal solution after one period from the analytic solution is again less than $10^{-5}$. Similarly to 
solutions belonging to the branch with $z=z_{2}$ the inverted cnoidal solutions belonging to the $z=z_{1}$ branch are resistant to small perturbations of the initial conditions. The motion is numerically stable for periods much longer than $T$, as well.

\section{Conclusions}

From our study the following conclusions can be drawn:

1. For extended Korteweg-de Vries equation exact solutions, both solitonic and periodic exist. These solutions have the same form as corresponding solutions of $\mathrm{KdV}$ equations but with coefficients altered.

2. KdV2 equation imposes severe limitations on its exact solutions. Physically relevant periodic solutions of $\mathrm{KdV} 2$ are related to two narrow intervals of the $m$ parameter. For $m$ very close to 1 , normal cnoidal waves are obtained. For $m$ very close to 0 , inverted cnoidal waves are found. This is a completely new result not present in the KdV case, since $\mathrm{KdV}$ fails for small $m$ values. In this case, however, wave profiles given by KdV2 solutions are not much different from a cosine function.

\section{References}

[1] G.G. Stokes, Camb. Trans. 8, 441 (1847). (Papers 1, 197).

[2] D.J. Korteweg, G. de Vries, Philos. Mag. 39, 422 (1895).

[3] T.B. Benjamin, J.L. Bona, J.J. Mahony, Philos. Trans. R. Soc. Lond. A 272, 47 (1972).

[4] J.L. Bona, W.G. Pritchard, L.R. Scott, Philos. Trans. R. Soc. Lond. A 302, 457 (1981).

[5] E. Infeld, G. Rowlands, A. Senatorski, Proc. R. Soc. Lond. A 455, 4363 (1999).

[6] T.R. Marchant, N.F. Smyth, J. Fluid Mech. 221, 263 (1990).

[7] T.R. Marchant, N.F. Smyth, IMA J. Appl. Math. 56, 157 (1996).

[8] G.I. Burde, A. Sergyeyev, J. Phys. A Math. Theor. 46, 075501 (2013).

[9] A. Karczewska, P. Rozmej, Ł. Rutkowski, Phys. Scr. 89, 054026 (2014).

[10] A. Karczewska, P. Rozmej, E. Infeld, Phys. Rev. E 90, 012907 (2014).
[11] A. Karczewska, P. Rozmej, E. Infeld, Phys. Rev. E 92, 053202 (2015).

[12] A. Sergyeyev, R. Vitolo, Nonl. Anal. Real World Appl. 32, 1 (2016).

[13] A. Karczewska, P. Rozmej, E. Infeld, G. Rowlands, Phys. Lett. A 381, 270 (2017).

[14] A.M. Wazwaz, Acta Phys. Pol. A 130, 679 (2016).

[15] K.R. Khusnutdinova, Y. Stepanyants, M. Tranter, Phys. Fluids 30, 022104 (2018).

[16] G. Rowlands, P. Rozmej, E. Infeld, A. Karczewska, Eur. Phys. J. E 40, 1 (2017).

[17] A.S. Fokas, Q.M. Liu, Phys. Rev. Lett. 77, 2347 (1996).

[18] H.R. Dullin, G.A. Gottwald, D.D. Holm, Phys. Rev. Lett. 87, 194501 (2001).

[19] Y. Kodama, Phys. Lett. A 107, 245 (1985); Phys. Lett. A 112, 193 (1985).

[20] Y. Hiraoka, Y. Kodama, Lect. Notes Phys. 767, 175 (2009).

[21] H.R. Dullin, G.A. Gottwald, D.D. Holm, Physica D 190, 1 (2004).

[22] R. Grimshaw, E. Pelinovsky, T. Talipova, Surv. Geophys. 28, 273 (2007).

[23] P. Rozmej, A. Karczewska, E. Infeld, Nonlin. Dyn. 91, 1085 (2018).

[24] P. Rozmej, A. Karczewska, Adv. Math. Phys. 2018 5095482 (2018).

[25] G.B. Whitham, Linear and Nonlinear Waves, Wiley, New York 1974.

[26] M.W. Dingemans, Water Wave Propagation over Uneven Bottoms. Part 2 - Nonlinear Wave Propagation, World Sci., 1997.

[27] A. Khare, A. Saxena, Phys. Lett. A 377, 2761 (2013).

[28] A. Khare, A. Saxena, J. Math. Phys. 55, 032701 (2014).

[29] A. Khare, A. Saxena, J. Math. Phys. 56, 032104 (2015).

[30] B. Abraham-Shrauner, Discr. Cont. Dyn. Syst. S 11 , 577 (2018).

[31] J.D. Fenton, J. Fluid Mech. 94, 129 (1979).

[32] J.D. Fenton, in: Ocean Engineering Science, The Sea, Eds. B. Le Méhauté, D.M Hanes, Wiley Interscience, 1990, p. 3.

[33] N.J. Zabusky, M.D. Kruskal, Phys. Rev. Lett. 15, 240 (1965).

[34] A. Karczewska, P. Rozmej, M. Szczeciński, B. Boguniewicz, Int. J. Appl. Math. Comp. Sci. 26, 555 (2016).

[35] A. Karczewska, M. Szczeciński, P. Rozmej, B. Boguniewicz, Comput. Meth. Sci. Techn. 22, 19 (2016). 